\section{Symmetry-induced formation of antivortices in mesoscopic superconductors}

\author{
Liviu F. Chibotaru*, Arnout Ceulemans ${ }^{\star}$, Vital Bruyndoncx $\dagger$ \\ \& Victor V. Moshchalkov $\dagger$
}

* Afdeling Kwantumchemie, Celestijnenlaan 200F, and $\dagger$ Laboratorium voor Vaste-Stoffysica en Magnetisme, Celestijnenlaan 200D, Katholieke Universiteit Leuven, B-3001 Leuven, Belgium

Recent progress in nanotechnology has stimulated interest in mesoscopic superconductors as components for quantum computing and cryoelectronics. The critical parameters for superconductivity (current and field) of a mesoscopic sample are determined by the pattern of vortices in it, which in turn is controlled by the symmetry imposed by the shape of the sample (see ref. 1 and references therein). Hitherto it has been unclear what happens when the number of vortices is not consistent with the natural symmetry. Here we show that additional vortexantivortex pairs nucleate spontaneously so as to preserve the symmetry of the sample. For example, in a square with three vortices, the spontaneously generated pair, along with the original three vortices, distribute themselves so that the four vortices sit in the four corners, with the antivortex in the centre. The measured superconducting phase boundary (of superconducting transition temperature $T_{\mathrm{c}}$ versus magnetic field strength) is in very good agreement with the calculations, giving direct experimental evidence for these symmetry-induced vortex-antivortex pairs. Vortex entry into the sample is also changed: vortices enter a square in fours, with antivortices generated to preserve the imposed vorticity. The symmetry-induced nucleation of antivortices is not restricted to superconductors, but should also apply to symmetrically confined superfluids and Bose-Einstein condensates.

The nucleation of superconductivity is normally analysed in terms of the linearized Ginzburg-Landau (LGL) equation ${ }^{2}$

$$
\frac{1}{2 m^{\star}}\left(-i \hbar \nabla-\frac{2 e}{c} \mathbf{A}\right)^{2} \psi=-\alpha \psi
$$

with $\mathbf{H}=\operatorname{rot} \mathbf{A}$ the applied magnetic field. Equation (1) looks like an ordinary Schrödinger equation for a free particle in an external magnetic field, where the 'wavefunction' $\psi$ stands for the complex superconducting order parameter and $\alpha=-\hbar^{2} / 2 m^{\star} \xi^{2}(T)$, with $\xi(T)$ the temperature-dependent coherence length. The superconducting boundary condition ${ }^{3}$ considerably complicates the solution of equation (1); we used a recently developed analytical gauge transformation, such that the vector potential A has no component normal to the sample boundaries of arbitrary regular polygons (L. F. C. et al., unpublished work). The lowest Landau level of equation (1) then gives the shift of the superconducting transition temperature $T_{\mathrm{c}}(H)$.

The solutions of the LGL equation for the square, shown in Fig. 1, are characterized by irreducible representations (irreps) $A, B, E_{-}$and $E_{+}$, with the characters $\exp (i n \pi / 2)$, for $n=0,2,-1$ or 1 under the fourfold rotation, respectively ${ }^{4}$. The ground Landau level shows an oscillatory cusplike behaviour as a function of flux, corresponding to a crossover of states belonging to different irreps (Fig. 1a).

Figure $1 \mathrm{~b}$ shows the comparison of the calculated and the measured phase boundary (open squares) for the mesoscopic Al square. The theoretical coloured curve is obtained from Fig. 1a, where the ground state level is selected for all flux values. The $T_{\mathrm{c}}(H)$ boundary is measured resistively, using an electronic feedback circuit. For experimental details, we refer to ref. 5. The agreement between the calculated lowest Landau level and the measured $T_{\mathrm{c}}(\Phi)$ is very good. We note that no fitting parameters were needed to match the cusp positions.

At the cusp positions on the phase boundary the vorticity $L$ changes by one, starting from zero (no fluxoids) at low magnetic fields. In the case of a disk $\left(C_{\infty}\right)$ the vorticity is just the orbital quantum number, $L$, defining the flux, $L \Phi_{0}$, carried by the giant
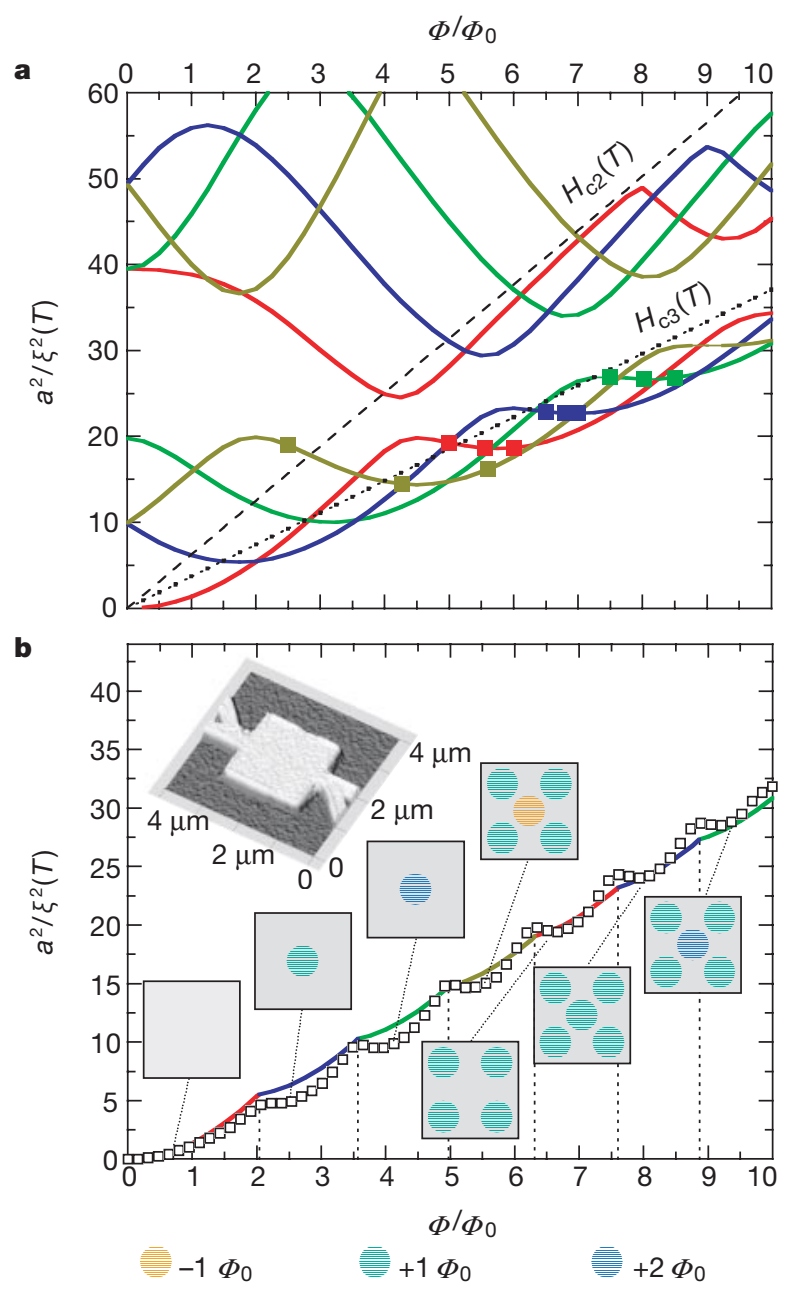

Figure 1 Calculated and measured superconducting $T_{\mathrm{c}}-H$ phase boundary for a square. a, Lower eigenvalues of the LGL equation for the mesoscopic square, as a function of magnetic flux $\Phi / \Phi_{0}$, with superconductor-vacuum boundary conditions. The different colours correspond to the four irreducible representations (irreps) $A$ (red), $B$ (green), $E_{+}$(dark blue) and $E_{-}$(dark yellow). Because of the discrete $C_{4}$ symmetry there is a 'repulsion' of the levels, giving a regular pattern of avoided crossings between levels belonging to the same irrep. The flux is defined as $\Phi=\mathrm{Ha}^{2}$, with a the side length of the square, and $H$ the applied magnetic field. The superconducting flux quantum

$\Phi_{0}=h / 2 e c$. On the vertical axis, the critical temperature $T_{\mathrm{c}}$ is linearly decreasing with increasing $a^{2} / \xi^{2}(T)$. The coloured squares on the lower lines indicate the values of $\Phi$ for which vortex patterns are shown in Figs $2-5$. The dashed straight line is the upper critical field $H_{\mathrm{c} 2}$ in a bulk type 2 superconductor, and $H_{\mathrm{c} 3}=1.69 H_{\mathrm{c} 2}$ (dotted straight line) is the surface critical field for a semi-infinite slab bordered by a straight superconductor-

vacuum interface. b, Comparison between the calculated (continuous coloured curve) and the measured $T_{c}(\Phi)$ phase boundary (open squares). The experimental data have been corrected for the presence of the measuring leads. A zero-temperature coherence length $\xi(0)=\xi(T) \sqrt{1-T / T_{\mathrm{c}}}=99 \mathrm{~nm}$ was used to obtain the best agreement. The zerofield critical temperature is $T_{\mathrm{c}}=1.36 \mathrm{~K}$. The top left inset shows the atomic force microscope image of the investigated $2 \times 2 \mu \mathrm{m}^{2}$ square. In the seven insets, the vortex structure in different regions of the phase diagram is shown schematically with coloured circles. In the range $5<\Phi / \Phi_{0}<6.3$, an antivortex is formed spontaneously at the centre of the square, coexisting with four $\Phi_{0}$-vortices along the diagonals. 
vortex ${ }^{6}$. For the square the rotational axis is of finite order $\left(C_{4}\right)$ and, therefore, the distribution of vortices in symmetry-consistent solutions, considered here, is not a priori evident. The seven insets in Fig. 1b show schematically the distribution of vortices, which are clearly different from the giant vortex states.

In the case of small $L$ values, vortices can occupy one central and four diagonal positions. In contrast to the diagonal vortices which always enclose a single quantum $\Phi_{0}$, the central vortex can have different winding numbers in order to conserve the total vorticity of a given state. The contribution of the two kinds of vortices (central + four diagonal) to the total winding number of the states shown in Fig. $1 \mathrm{~b}$ is given by

$$
L=n+4 m
$$

where $n=0,1,2$ or -1 and $m=0$ or 1 .

First, the nature of the central vortex changes, whenever vorticity is changed by one. Thus, the central vortex is absent in the first state, is a $\Phi_{0}$-vortex in the second state, is a giant vortex in the third state and is an antivortex (the winding number is negative) in the fourth state (see Fig. 1b). Second, the sequence of winding numbers of the central vortex $(-1,0,1,2)$ is periodically repeated when going to the right of the phase diagram. Because the kinetic energy of a vortex is proportional to $L^{2}$, the system prefers to split the giant vortex into a sum of smaller vortices ${ }^{7}$ if there are no special symmetry restrictions. This explains why only four numbers mentioned above appear as winding numbers for the central vortex. On the other hand, the formation of antivortices is dictated completely by the discrete symmetry. Indeed, in the state with $L=3$, one cannot distribute three $\Phi_{0}$-vortices on the square keeping the symmetry. The dilemma is solved by having four $\Phi_{0}$-vortices and adding one antivortex in the centre. This is also the lowest energy state

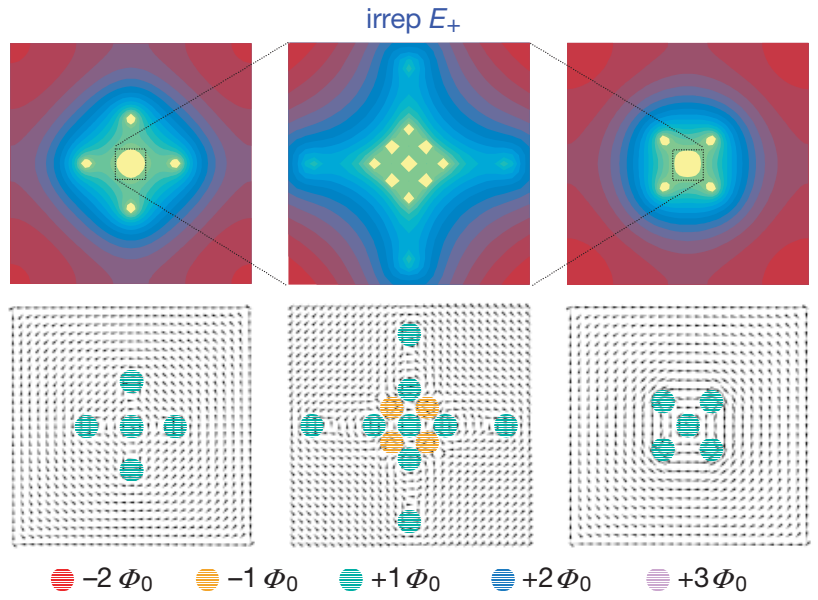

Figure 2 Vortex entry into a square for applied magnetic fields generating five flux quanta. The symmetry of the corresponding states is $E_{+}$(blue curves in Fig. 1). The top panels show the density of the order parameter $|\psi|^{2}$ for the initial, intermediate and final stages corresponding to the $\Phi$ values marked by the squares (from left to right) in Fig. 1a. The highest density is shown in red. The lowest $|\psi|^{2}$ values are coloured yellow, indicating the positions of vortices and antivortices. The lower three panels show the phase gradient of the order parameter, corresponding to the above contour plots. The gradient is represented by arrows with appropriately scaled lengths. The coloured filled circles in the lower three panels relate each vortex to its winding number (see below). Here, in the middle panels the central region of the square was magnified 16 times. Stage one (on the left) shows the four 'lateral' vortices approaching the central vortex from the middles of the square's sides. At the transition state (in the centre), the central region suddenly changes into a chequerboard-like pattern of vortices and antivortices. In a subsequent step (not shown here) the vortices from the four pairs of lateral vortices approach each other and suddenly 'rotate' by $90^{\circ}$. As the flux further increases, the antivortices and the 'rotated' $\Phi_{0}$-vortices move towards the corners and eventually merge to form single diagonal $\Phi_{0}$-vortices (on the right). (compare with the fourth state in Fig. 1b).

Spontaneous generation of antivortices also controls the flux penetration into mesoscopic superconductors. Our theoretical analysis shows that in regular polygons with $N$ edges the flux enters always by $N \Phi_{0}$-vortices through the edge centres, because these are the symmetry points with the lowest values of $|\psi|$ on the borders. Increasing the field further, it is energetically favourable for these vortices to reorient towards the corners of the polygon (Fig. $1 b$ ), thus paving the way for the entrance of the next set of $N \Phi_{0^{-}}$ vortices. However, such a reorientation cannot be performed by a continuous rotation of the vortex patterns, as that would violate the imposed symmetry. Therefore, the formation of additional antivortices and vortices turns out to be indispensable.

This is further illustrated for the square. Three states in the evolution of the vortex patterns - entrance of vortices (initial), the transient state (intermediate), and the formation of diagonal vortices (final)-are shown in Figs $2-5$ for the four irreps. The dynamics of this transformation differs dramatically for states of different symmetry. Thus, in the case of irreps $E_{+}$and $A$ four vortices and four antivortices arise in the intermediate state. For the other two irreps the intermediate state is associated with the change of the winding number of the central vortex: the giant $2 \Phi_{0}$-vortex decays into a giant antivortex $-2 \Phi_{0}$ and four diagonal $\Phi_{0}$-vortices in the case of irrep $B$, whereas for the irrep $E_{-}$the central $\Phi_{0}$-antivortex is transformed into a giant $3 \Phi_{0}$-vortex by absorbing the four lateral $\Phi_{0}$-vortices. For direct visualization of these unusual vortex patterns (Figs 1-5), local vortex-imaging techniques, such as scanning Hall probe $^{8,9}$, scanning tunnelling ${ }^{10,11}$, magnetic force ${ }^{12}$, and Lorentz microscopy ${ }^{13}$ are very promising.

The appearance of symmetry-induced antivortices remains valid for all other superconducting polygons (triangle, pentagon, and so on). Our results might also be applicable to large antidot arrays, where the spontaneous generation of antivortices could provide conditions for stronger vortex pinning. The spontaneous generation of antivortices is clearly a fundamental property of symmetrically confined vortex matter in general. Our findings are applicable not only to superconductors, but also to superfluids $\left({ }^{4} \mathrm{He}\right.$ and $\left.{ }^{3} \mathrm{He}\right)$ and Bose-Einstein condensates. Superfluids rotated in a triangular or

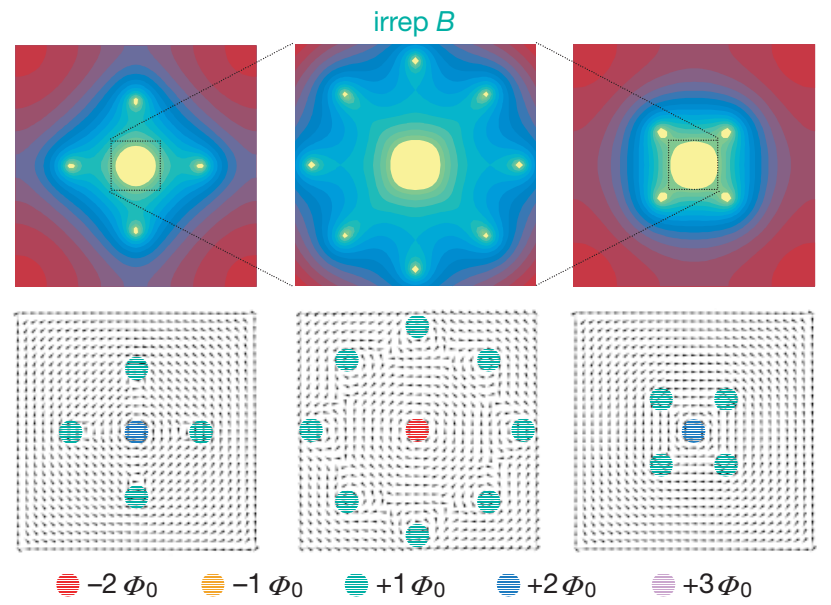

Figure 3 Vortex entry into a square for applied magnetic fields generating six flux quanta. The symmetry of the corresponding states is $B$ (green curves in Fig. 1). The same colour conventions as in Fig. 2 were used. The middle plots correspond to the central region of the square magnified 5 times. The reorientation mechanism in this case involves the transformation of the central $2 \Phi_{0}$ giant vortex (on the left) into a giant antivortex ( $-2 \Phi_{0}$ vortex) and four diagonal $\Phi_{0}$-vortices (central panels). Under this transformation the total vorticity remains invariant: $8 \times \Phi_{0}-1 \times 2 \Phi_{0}=6 \Phi_{0}$. While the diagonal vortices move to the corners, the lateral $\Phi_{0}$-vortices further approach the centre and are absorbed by the central antivortex, restoring in this way the initial $2 \Phi_{0}$ giant vortex, characteristic for the irrep $B$. 
square vessel would also generate antivortices in order to comply with the imposed symmetry. By proper arrangement of the laser fields, the vortex patterns in Bose-Einstein condensates confined by triangular or square traps could also reveal symmetry-induced antivortices.

Our symmetry-conserving results for a triangle form a natural generalization to superconducting boundary conditions of the quantum-mechanical problem of a "particle in an equilateral triangle" ${ }^{14}$. An intriguing correspondence can be drawn between

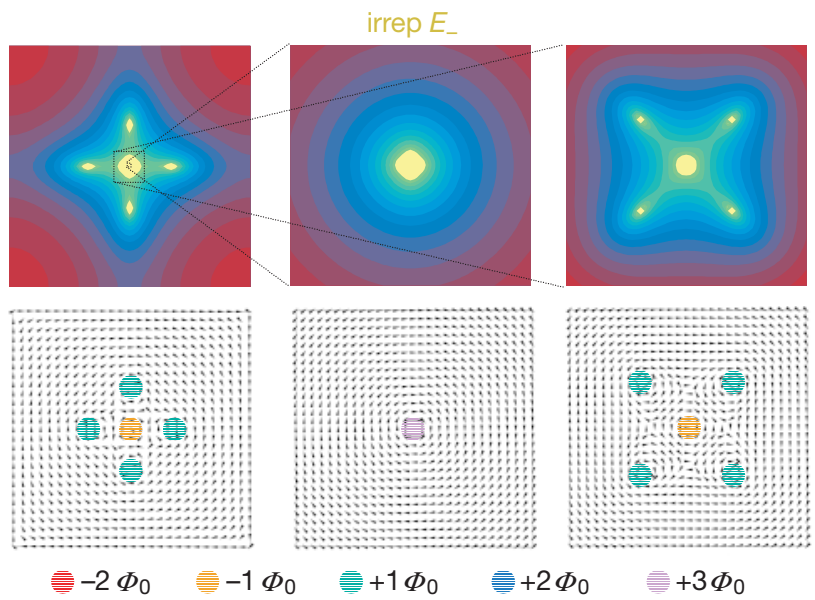

Figure 4 Vortex entry into a square for applied magnetic fields generating three flux quanta. The symmetry of the corresponding states is $E_{-}$(dark yellow curves in Fig. 1). The same colour conventions as in Fig. 2 were used. The central region of the square is magnified 64 times, for the middle plots, and 8 times for the plots on the right. In this mode, the lateral $\Phi_{0}$-vortices approach and merge with the central antivortex to form a giant $3 \Phi_{0}$-vortex. With increasing field, the $3 \Phi_{0}$-vortex will split into four diagonal $\Phi_{0^{-}}$ vortices and the initial single antivortex (on the right). In order to be able to see the four separate $\Phi_{0}$-vortices, the panels on the right had to be magnified. This is owing to the strong attraction of the $\Phi_{0}$-vortices to the central antivortex. For the same reason, the spacing between the three dark yellow squares in Fig. 1a is large, as compared to the other irreps.

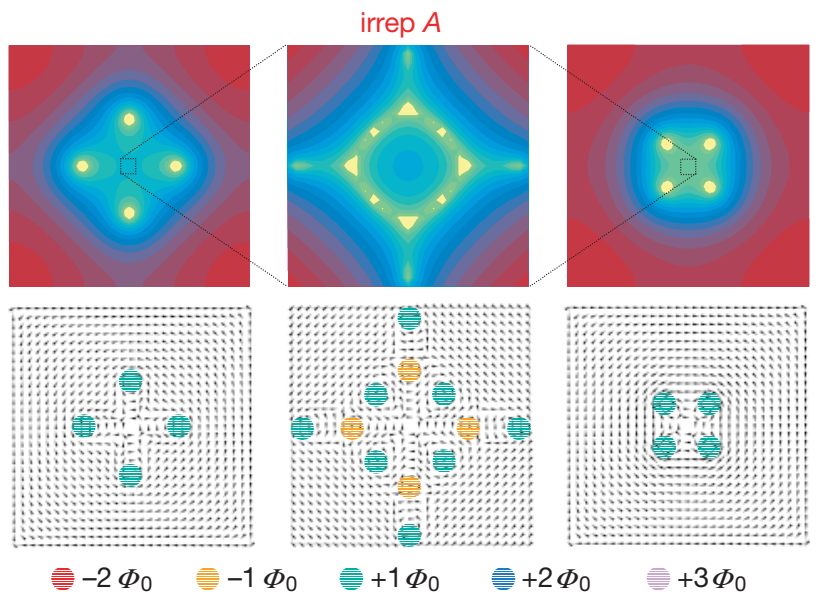

Figure $\mathbf{5}$ Vortex entry into a square for applied magnetic fields generating four flux quanta. The symmetry of the corresponding states is $A$ (red curves in Fig. 1). The same colour conventions as in Fig. 2 were used. The middle plots correspond to the central region of the square magnified 16 times. In the initial stage (left), there is no central vortex in this case. The approach of the lateral $\Phi_{0}$-vortices induces the creation of a central vortex-antivortex pattern (central panels), which is however rotated by $45^{\circ}$, as compared to Fig. 2. At higher flux (on the right), the lateral vortices and antivortices will annihilate, leaving four diagonal $\Phi_{0}$-vortices which move towards the corners. the eigenstates in the triangle and families of leptons (electrons or muons) and quarks.

1. Moshchalkov, V. V. et al. in Handbook of Nanostructured Materials and Nanotechnology (ed. Nalwa, H. S.) Vol. 3, Ch. 9, 451-525 (Academic, San Diego, 1999).

2. de Gennes, P.-G. Superconductivity of Metals and Alloys (Benjamin, New York, 1966).

3. Saint-James, D. \& de Gennes, P.-G. Onset of superconductivity in decreasing fields. Phys. Lett. 7, 306308 (1963).

4. Landau, L. D. \& Lifshitz, E M. Quantum Mechanics 2nd edn (Pergamon, Oxford, 1975).

5. Bruyndoncx, V., Strunk, C., Moshchalkov, V. V., Van Haesendonck, C. \& Bruynseraede, Y. Fluxoid quantization effects in superconducting mesoscopic Al multiloop structures. Europhys. Lett. 36, 449454 (1996).

6. Saint-James, D. Etude du champ critique $H_{\mathrm{c} 3}$ dans une géometrie cylindrique. Phys. Lett. 15, 13-15 (1965).

7. Abrikosov, A. A. Fundamentals of the Theory of Metals (North-Holland, Amsterdam, 1988).

8. Chang, A. M. et al. Scanning Hall probe microscopy. Appl. Phys. Lett. 61, 1974-1976 (1992).

9. Oral, A., Bending, S. J. \& Henini, M. Real-time scanning Hall probe microscopy. Appl. Phys. Lett. 69, 1324-1326 (1996).

10. Hess, H. F., Robinson, R. B., Dynes, R. C., Valles, J. M. Jr \& Waszcak, J. V. Scanning-tunnelingmicroscope observation of the Abrikosov flux lattice and the density of states near and inside a fluxoid. Phys. Rev. Lett. 62, 214-216 (1989).

11. Maggio-Aprile, I., Renner, Ch., Erb, A., Walker, E. \& Fischer, O. Direct vortex lattice imaging and tunneling spectroscopy of flux lines on $\mathrm{YBa}_{2} \mathrm{Cu}_{3} \mathrm{O}_{7-\delta}$. Phys. Rev. Lett. 75, 2754-2757 (1995).

12. Moser, A. et al. Observation of single vortices condensed into a vortex-glass phase by magnetic force microscopy. Phys. Rev. Lett. 74, 1847-1850 (1995).

13. Harada, K. et al. Direct observation of vortex dynamics in superconducting films with regular arrays of defects. Science 274, 1167-1169 (1996).

14. Li, W.-K. \& Blinder, S. M. Solution of the Schrödinger equation for a particle in an equilateral triangle. J. Math. Phys. 26, 2784-2786 (1985).

\section{Acknowledgements}

The work was supported by the ESF programme VORTEX, Concerted Action Scheme (GOA) of the Flemish Government and by the Fonds voor Wetenschappelijk Onderzoek Vlaanderen (FWO). We would like to thank L. Van Look for taking the atomic force microscope micrograph.

Correspondence and requests for materials should be addressed to V.V.M. (e-mail: victor.moshchalkov@fys.kuleuven.ac.be).

\section{Flexible filaments in a flowing soap film as a model for one-dimensional flags in a two-dimensional wind}

\author{
Jun Zhang ${ }^{\star} \dagger$, Stephen Childress ${ }^{\star}$, Albert Libchaber $\dagger$ \& Michael Shelley \\ * Applied Mathematics Laboratory, Courant Institute, New York University, \\ New York 10012, USA \\ $\dagger$ Center for Studies in Physics and Biology, Rockefeller University, \\ New York 10021, USA
}

The dynamics of swimming fish and flapping flags involves a complicated interaction of their deformable shapes with the surrounding fluid flow. Even in the passive case of a flag, the flag exerts forces on the fluid through its own inertia and elastic responses, and is likewise acted on by hydrodynamic pressure and drag. But such couplings are not well understood. Here we study these interactions experimentally, using an analogous system of flexible filaments in flowing soap films. We find that, for a single filament (or 'flag') held at its upstream end and otherwise unconstrained, there are two distinct, stable dynamical states. The first is a stretched-straight state: the filament is immobile and aligned in the flow direction. The existence of this state seems to refute the common belief that a flag is always unstable and will flap $^{1,2}$. The second is a flapping state: the filament executes a sinuous motion in a manner akin to the flapping of a flag in the wind. We study further the hydrodynamically coupled interaction between two such filaments, and demonstrate the existence of four different dynamical states. 